\title{
Design of a Series Elastic- and Bowdencable-based actuation system for use as torque-actuator in exoskeleton-type training
}

\author{
J.F. Veneman, R. Ekkelenkamp, R. Kruidhof, F.C.T. van der Helm, H. van der Kooij \\ Biomechanical Engineering, BMTI, University of Twente, \\ P.O. Box 217, 7500 AE Enschede, The Netherlands

\section{j.f.veneman@utwente.nl}

\begin{abstract}
Common actuators have important drawbacks for use in an exoskeleton type of rehabilitation (training) robot. Either the actuators are heavy, complex or poor torque sources. A new actuation system is proposed and tested that combines a lightweight joint and a simple structure with adequate torque source quality. It consists of a servomotor, a flexible Bowden cable transmission, and a force feedback loop based on a series elastic element. Measurements show that performance is sufficient for use in a gait rehabilitation robot.
\end{abstract}

\section{INTRODUCTION}

$\mathrm{W}_{\mathrm{c}}^{\mathrm{e}}$ e are developing a LOwer-extremity Powered ExoSkeleton (LOPES), a gait training robot. The target group consists of people who (partly) lost their walking ability due to a stroke (CVA). The robot is built for use in combination with a treadmill. As a 'robotic therapist' it is meant to make rehabilitation more effective for patients and less demanding for therapists [1,2].

The main functionality of LOPES will be replacing physiotherapists in their mechanical interaction with patients, while leaving clinical decisions to their judgment. This mechanical interaction mainly intends assistance in leg movements in the forward direction and in keeping lateral balance.

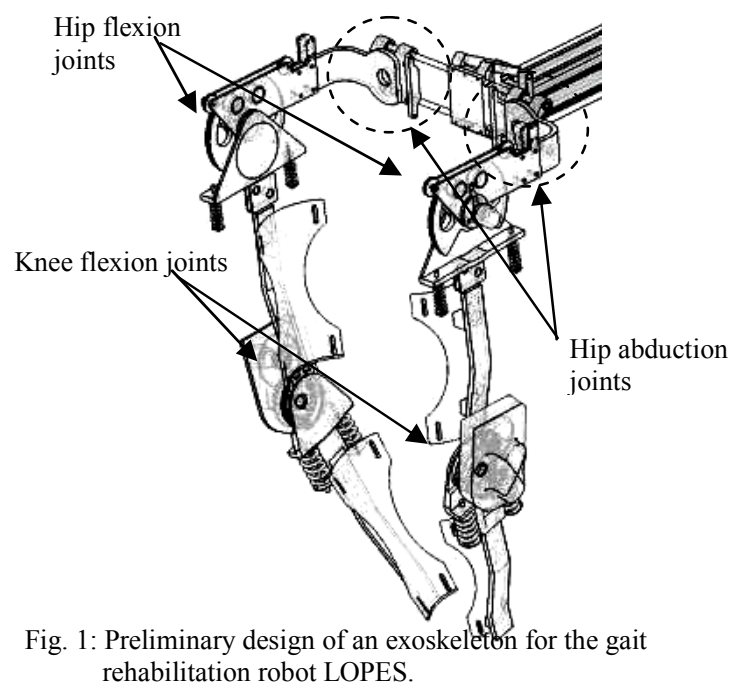

Within the LOPES project, it has been decided to realize this by connecting the limbs of the patient to an 'exoskeleton' so that robot and patient move in parallel (Fig. 1). The exoskeleton is actuated in order to realize supportive actions and preventing fail mechanisms in walking, such as: assuring enough foot clearance, stabilizing the knee, shifting the weight, et cetera [3]. A general aim is to let the patient walk as unhindered as possible, while offering a minimum of necessary support and a safe training environment. This constitutes the main difference between LOPES and other gait-trainers. Those are either position controlled devices that overrule the patient and/or allow only limited motions due to a limited number of degrees of freedom, and/or are not fully actuated (or are not yet functional)[2,4].

Two 'extreme' ideal modes can be defined, which span the full range of therapeutic interventions demanded in the LOPES project. In one ideal mode, referred to as 'robot in charge', the robot should be able to enforce a desired walking pattern (high impedance), defined by parameters like walking speed and step-length. In the other ideal mode, referred to as 'patient in charge' the robot should be able to follow the walking patient while hardly hindering him or her (low impedance). An intelligent controller then would be able to vary the actual robot behavior between these maximal and minimal impedance modes.

In the 'robot in charge'-mode it is important that the robot has enough bandwidth and power to realize the desired positioning performance with the desired stiffness (impedance). In the 'patient in charge'-mode it is important that interaction forces between exoskeleton and human are controlled towards zero; in other words that the perceived impedance of the robot is low. In LOPES this will be realized using an impedance controller, as is often applied in haptic interfaces [5]. This implies specific demands for the actuators in the robot. They should:

- be 'pure' (low impedance) force sources

- add little weight and friction to the moving robot construction in any degree of freedom

- be as safe as possible, even in case of failure

- allow fast adjustment to the individual patients' sizes

- be powerful enough for the 'robot in charge' task.

More specific, it is required that the actuators should be able to modulate their output force with $12 \mathrm{~Hz}$ for small forces, and $4 \mathrm{~Hz}$ for the full force range. Maximum torques differ per joint, and range from 25 to $60 \mathrm{Nm}$. These demands were based on study of the human gait cycle and the human therapist. The resulting bandwidths are typically slightly lower, and the forces typically higher than in the specifications of common haptic (kinaesthetic) devices 
which are intended to display a virtual object, but not to assist. This results in a different choice of actuators.

Actuators usually selected for kinaesthetic devices are either heavy (like direct drive electro-motors) or poor force actuators (like geared DC motors), or suffer from poor suitability for a rehabilitation setting due complex non-linear behavior or lack of safety (like pneumatic muscles or cylinders). The alternative to use (e.g. cable) transmissions generally hinders fast adjustment of exoskeleton sizes.

In this abstract an actuator will be presented that avoids these disadvantages by combining a flexible cable based transmission with a spring based feedback force control loop. This allows for flexibly detaching the actuator from the robot frame, while achieving appropriate force control performance. First the construction will be presented, followed by measurements that show the achieved performance.

\section{CONSTRUCtive SOLUTION}

The global lay-out of the developed actuator system is depicted in Fig. 2. The basic idea was to detach the actual motor from the robot frame by the use of flexible Bowden cables so that the exoskeleton legs can move unhindered, while little weight is added to the robot construction.

The actuator system is constructed as a rotating joint, which is a torque source. Such joints can be integrated in the exoskeleton (Fig. 1) as for example hip and knee joints.

Double cables were needed because expected tensions were larger than would be allowed for single cables. Both cables are continuous units. In case a cable breaks, it will loose its tension and no safety threat will occur due unidirectional force exercise. Because Bowden-cables introduce orientation-, speed- and tension-dependent friction, friction compensation is needed. The angles of the curves in the cable and their radii are the main determinants of the actual friction. Also wear and tension of the cables are important determinants. Because these parameters are hardly observable, it is impossible to compensate the friction properly with feedforward control. Acceptable compensation may be achieved by introducing a feedback force control loop. This requires a force measurement located after the cable transmission. We chose to use springs for force measuring. A spring can be considered as a compliant force

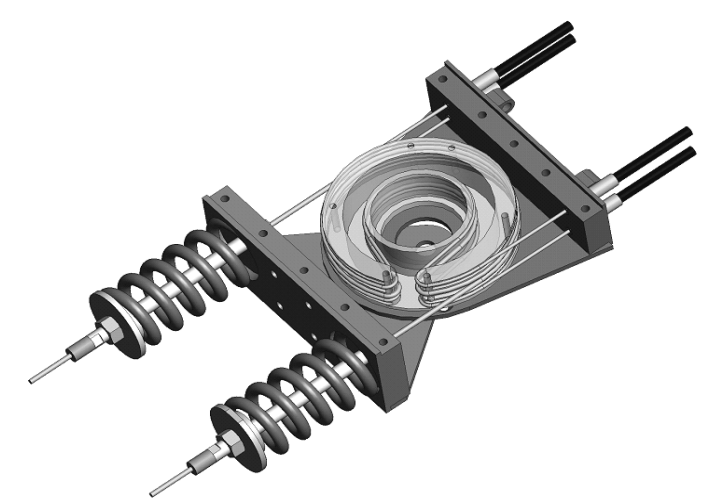

sensor. It also allows treating the force control loop as a position control, because the spring length is proportional to the force output. A relative high compliance in the force sensor allows for higher control gains in the feedback spring length control loop.

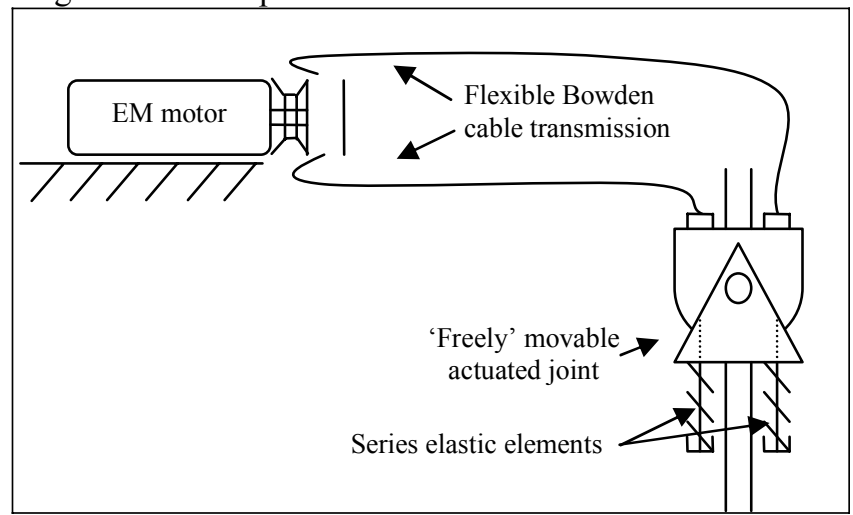

Fig. 2: Global lay-out of the proposed actuator system. The actuated joint is lightweight as the motor is placed on the fixed world.

This way a better force control performance and actuator impact resistance can be achieved. The concept is similar to Series Elastic Actuation (SEA), treated extensively in [6]. This theoretical framework has to be only slightly adapted to be applicable on the actuator presented here. The differences with that SEA are that we constructed a rotational joint instead of a linear, integrated the actuator with the robotjoint and added a Bowden cable transmission. The two pretensioned compression springs function as a 'series elastic' torsion spring placed in-between the actuated disk and the actual joint.

The Bowden cable transmission might negatively influence the bandwidth of the actuator compared to common SEA, as it introduces complex friction, compliance and play into the position control loop. On the other hand, it is possible to select a heavier motor, as the weight is of no importance in this setting and a larger motor mass will increase the bandwidth of the actuator [7].

Basic design parameters, besides the choice of motor and gearing, are the joint-disk diameter and the stiffness of the springs. The diameter is a compromise of a small or large size of the joint versus low or high tension (thus friction) in

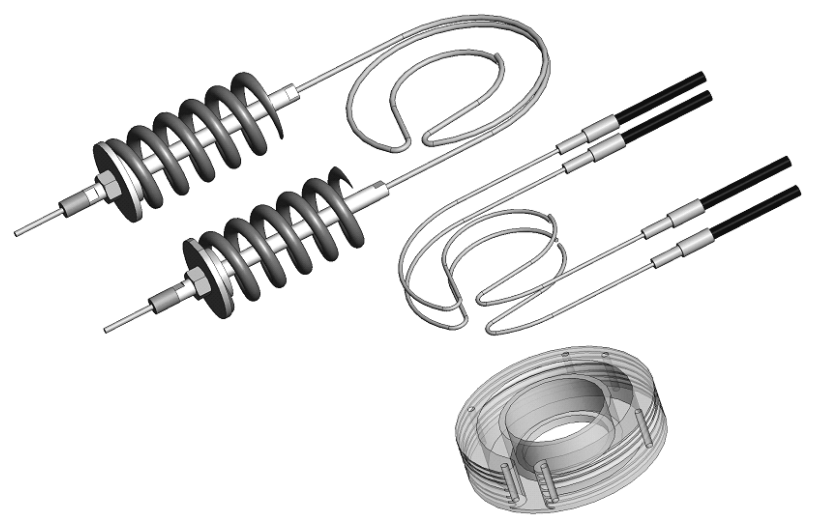

Fig. 3: The picture shows the actuated joint, the other side (not visible) of the Bowden cables is connected to a second disk driven by a servo motor. The cable with the two springs is the connection between the actuated disk and the joint output axis. 
the cables. The stiffness is a compromise of a high force control bandwidth versus minimal endpoint impedance combined with optimal non-linear (cable-)friction compensation [7].

Performance measurements will now be presented to show that this actuator suffices for the described application. Also the influence of extreme Bowden cable bending will be shown. The proposed actuator was designed and built to function as a knee joint. With this set-up all presented measurements were carried out. It has an approximate peak torque output of $30 \mathrm{Nm}$. Four Bowden cables of $1.5 \mathrm{~m}$ each were used. An LVDT sensor was used for spring length measurement.

\section{Measurements AND Results}

Measurements were done to determine the performance of the current configuration. Of course performance depends on many small constructive and controller decisions, so the outcome should be interpreted as a mere indication of the achievable performance with the presented type of actuation. The joint is as much as possible used in the way it will be used in the final design of the rehabilitation robot.

The optimal course of a Bowden cable would be straight, as bending introduces friction and play. A realistic standard situation was defined in which the Bowden cables are bent over $90^{\circ}$ with a radius of $0.8 \mathrm{~m}$. Additional measurements were done with cables bent over $180^{\circ}$ with a radius of $0.4 \mathrm{~m}$. Such a configuration should be avoided during operation, but is meant as a worst case example.

\section{A. Preliminary check: measurement accuracy}

As the springs in the joint are used for force measurement in the control loop, a first performance check is how well the spring-deflection reflects the actual force output. To check this, the joint was fixed on both sides, on one side via a force sensor. From the force sensor measurements the joint torque was calculated and this was compared to the force output based on the spring deflection. A ramp signal was used as reference input. A representative measurement (Fig. 4) shows that the measurement based on the spring deflection is acceptably representing the actual torque output.

\section{B. First performance check: force control bandwidth}

A first performance check, related to the 'robot in charge'-mode, is the force bandwidth of the actuator. An open loop bode plot (Fig.5) of the actuator behavior is given to show the system limitations. The open loop is defined here as the transfer from the control command (the reference value for the motor torque) to the actuator torque output.

A closed loop bode (Fig.6) is given to show what may be achieved with the actual system and a standard Ziegler Nichols tuned PID controller.

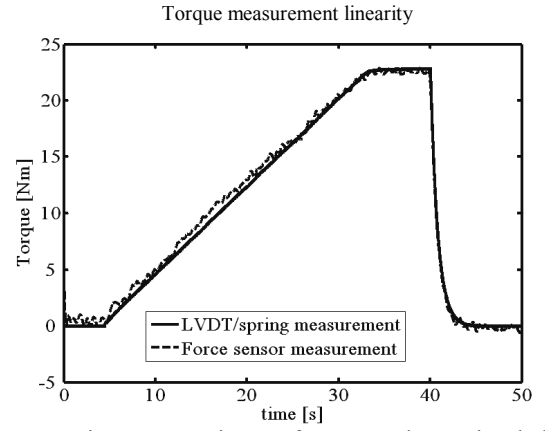

Fig. 4: representative comparison of torque determined from an lvdt measurement of spring deflection versus torque determined from a force sensor measurement. The reference for the torque was a ramp signal, controlled on the spring measurement.

All experiments described in this abstract used this same controller. The closed loop is defined here as the transfer from reference torque command to actuator torque output using the feedback control.

In these bode plots also the effect of the excessive Bowden cable bending is shown, as this worsens the force control performance (Fig.5,7). These plots imply that the smaller the control command (motor torque command) and the larger the bending radius, the bigger the relative influence of (static) friction components becomes.
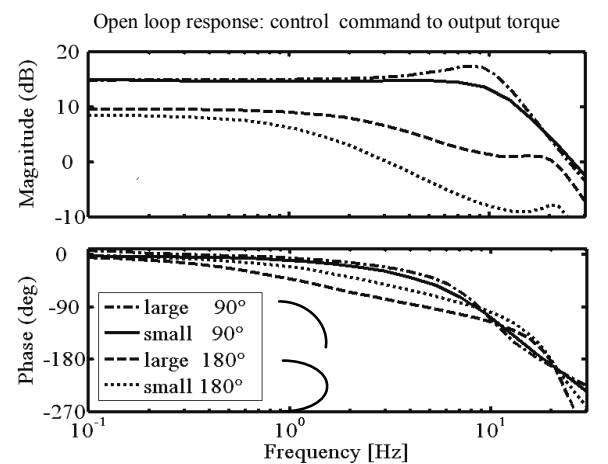

Fig. 5: Open loop response of the actuator. Bode plot of the transfer from input control command to output joint torque. The RMS amplitude of the input signal [small,large] and the course of the Bowden cables have been varied $\left[90^{\circ}, 180^{\circ}\right]$.

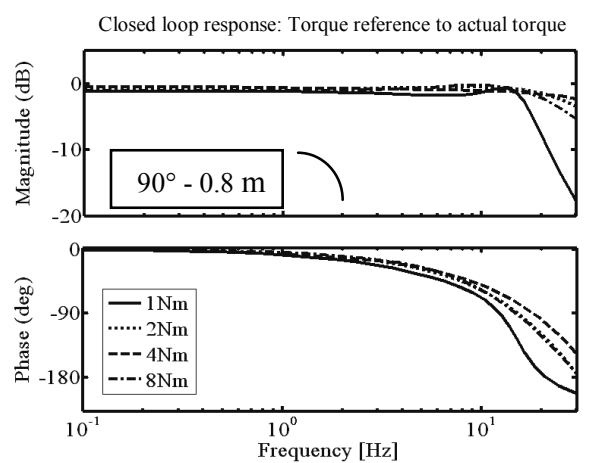

Fig. 6: Bode plot of the closed loop transfer from reference torque to actual torque. The RMS amplitude of the reference was varied. Here the orientation was $90^{\circ}$ with a bending radius of $0.8 \mathrm{~m}$. 


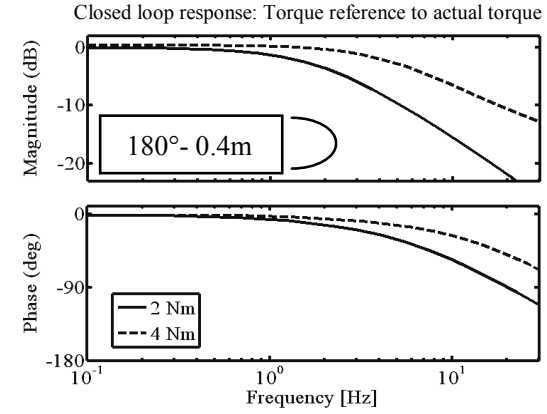

Fig. 7: Bode plot of the closed loop transfer from reference torque to actual torque. The RMS amplitude of the reference was varied. Here the orientation was $180^{\circ}$ with a bending radius of $0.4 \mathrm{~m}$.

It can be seen that the controlled bandwidth of the actuator is about $20 \mathrm{~Hz}$ for the measured range of torques. For small torques the transfer falls off at lower frequencies, although still above $12 \mathrm{~Hz}$. This can be explained by the static friction in the Bowden cable transmission, which hinders the cable motion in case of small control values.

The open loop bode (Fig.5) shows that excessive bending of the cables $\left(180^{\circ}\right)$ increases friction dramatically. It can be concluded that the 'bending range' of the cables is limited; the total bending should not become too big, nor the bending radius too small. This can be prevented by a proper design.

\section{Second performance check: zero-impedance control}

The second performance check, related to the 'patient in charge'-mode is the quality of 'zero-impedance' control. This performance is checked by imposing a position trajectory upon the output axis of the joint by hand, and then measuring the reaction force of the actuated joint. The spring length was used as force measurement. The transfer from joint angle to actuator torque then is the mentioned impedance. The impedance was measured both in the controlled and uncontrolled situation, to show the improvement obtained by using a force feedback loop. The measurements for the controlled situation were done using the same force control settings as above, while using a zero reference force. It appeared that the power spectrum of the hand-perturbations ranged from about 0.7 to $4 \mathrm{~Hz}$. The results are presented in a bode plot (Fig.8).

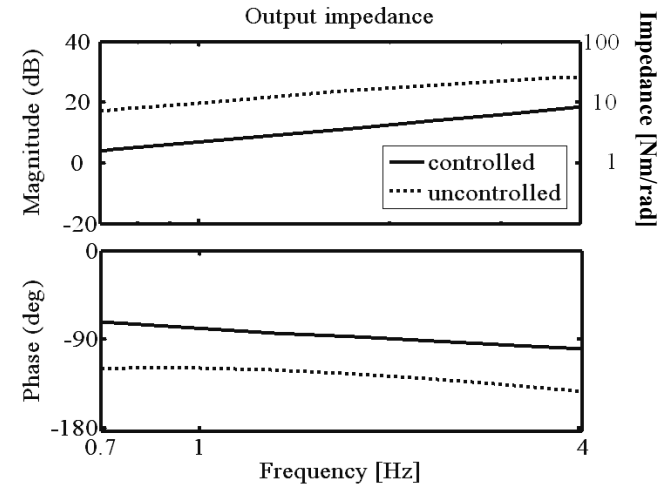

Fig. 8: Bode plot of the impedance identifications of several measurement-series in both controlled and uncontrolled situations. The input motion disturbance signal was applied by hand. Only the frequency range with appropriate coherence is shown.
From this figure it can be concluded that a considerable reduction $(10-13 \mathrm{~dB}$; factor $3-4.6)$ of impedance can be realized with feedback control in the frequency range of application. The noticeable effects of cable stick forces on the output force are also strongly reduced. Tests with a walking subject connected to this joint confirmed that the controlled impedance was low enough to experience unhindered lower leg motion.

\section{CONCLUSION}

A new Bowden-cable-based series elastic actuation system was proposed for use in exoskeleton-type rehabilitation robots. A robot joint containing this actuation system was constructed. It was shown that the performance demands of realizing a safe, lightweight, adjustable and powerful torque source were met. A force bandwidth of up to $20 \mathrm{~Hz}$ appeared feasible using suitable cable courses. This is considered adequate to control human gait. The joint appeared also suitable for low impedance control in an exoskeleton leg to facilitate unhindered motion. This implies that a robot using these joints would be able to be used in both a 'robot-in-charge' as well as in a 'patient in charge' mode.

Future work includes determining the influence of altering spring stiffness and motor size. Also thicker Bowden cables will be used to allow the use of one pair of cables instead of two. Finally the controller has to be optimized so it can deal better with the non-linear effects like varying damping, and static friction inside the position control loop.

\section{ACKNOWLEDGEMENT}

This research is supported by the NWO (vernieuwingsimpuls 2001, granted to dr. H. van der Kooij) and by the institute for Biomedical Technology.

\section{REFERENCES}

[1] H.I.Krebs, PhD; B.T.Volpe, MD; M.L.Aisen, MD; N.Hogan, PhD, "Increasing productivity and quality of care: Robot-aided neurorehabilitation", Journal of Rehabilitation Research and Development Vol. 37 No. 6, November/December 2000

[2] D.J.Reinkensmeyer, J.L.Emken, and S.C.Cramer, "Robotics, motor learning, and neurologic recovery," Annual Review of Biomedical Engineering, vol. 6, pp. 497-525, 2004.

[3] R.Ekkelenkamp, J.Veneman, H.van der Kooij, "LOPES: Selective control of gait functions during the gait rehabilitation of CVA patients", Proceedings of the ICORR 2005, to be published, 2005.

[4] S.Hesse, H.Schmidt, C.Werner, and A.Bardeleben, "Upper and lower extremity robotic devices for rehabilitation and for studying motor control," Current Opinion in Neurology, vol.16, pp.705-710, 2003.

[5] R.J.Adams and B.Hannaford, "Control law design for haptic interfaces to virtual reality," Ieee Transactions on Control Systems Technology, vol. 10, pp. 3-13, 2002.

[6] D.W.Robinson, "Design and Analysis of Series Elasticity in Closed loop Actuator Force Control", Ph.D. dissertation, Massachusetts Institute of Technology (MIT), 2000.

[7] D.W.Robinson, J.E.Pratt, D.J.Paluska, and G.A.Pratt, "Series elastic actuator development for a biomimetic robot", IEEE/ASME International Conference on Advanced Intelligent Mechatronics, 1999. 